\title{
Convex Corson compacta and Radon measures
}

\author{
by \\ Grzegorz Plebanek (Wrocław)
}

Abstract. Assuming the continuum hypothesis, we show that

(i) there is a compact convex subset $L$ of $\Sigma\left(\mathbb{R}^{\omega_{1}}\right)$, and a probability Radon measure on $L$ which has no separable support;

(ii) there is a Corson compact space $K$, and a convex weak ${ }^{*}$-compact set $M$ of Radon probability measures on $K$ which has no $G_{\delta}$-points.

1. Introduction. In an important paper [1], S. Argyros, S. Mercourakis and S. Negrepontis proved several results on the structure of Corson compact spaces and Banach spaces of continuous functions on such compacta. Section 3 of [1] is devoted to the study of Corson compact spaces with property (M), i.e. spaces on which every Radon measure has a separable support. In particular, the following statement may be found there:

(*) If $L$ is a convex compact subset of $\Sigma\left(\mathbb{R}^{\kappa}\right)$ then $L$ has property $(\mathrm{M})$.

Recall that it follows from Martin's axiom and the negation of the continuum hypothesis that every Corson compact space has property (M) (see [13] or Section 2 below), so the point here is that the convexity assumption enables one to prove the claim without additional set-theoretic assumptions.

As observed by O. Kalenda, the proof of $(*)$ given on p. 215 of [1] contains a gap, namely the final statement of that proof is not correct. Since that difficulty seemed to be hard to overcome, O. Kalenda asked if $(*)$ was true at all (more precisely, if $(*)$ is provable in $\mathrm{ZFC}$ ).

It was noted by Todorčević (see [21, Lemma 9.5]) that $(*)$ holds under the additional assumption that $L$ is contained in $\Sigma\left(\mathbb{R}_{+}^{\kappa}\right)$. Using similar methods we prove below that every Radon measure of countable type defined on a compact convex subset of $\Sigma\left(\mathbb{R}^{\kappa}\right)$ has a separable support (Theorem 2.2). It turns out, however, that $(*)$ need not hold: Assuming the continuum

2000 Mathematics Subject Classification: Primary 28C15; Secondary 46E27.

Partially supported by KBN grant 5 P03A 037 20. This research was done while the author held a visiting position at the Institute of Mathematics, Polish Academy of Sciences. 
hypothesis $(\mathrm{CH})$ we construct a convex compact set $L \subseteq \Sigma\left(\mathbb{R}^{\omega_{1}}\right)$ with a Radon measure which has no separable support (Theorem 4.2).

The paper is organized as follows. In Section 2 we recall basic facts concerning Radon measures and Corson compact spaces, and give a short account of (essentially known) results related to the question of whether every Radon measure defined on a Corson compact space has a separable support. In Section 3 we present two $\mathrm{CH}$ examples of 0-dimensional Corson compact spaces with measures, both resulting from various algebras of sets. The first one is used in the proof of the main result of Section 4. The other is a Corson compact space $K$ such that there is a nonempty weak*-compact convex subset of $C(K)^{*}$ having no $G_{\delta}$-points. This answers a problem posed in [16, Question 2, p. 517]. Section 4 contains a construction of a $\mathrm{CH}$ counterexample to $(*)$ mentioned above, and a related result (due to O. Kalenda) on Banach spaces with a Corson compact dual unit ball.

The author is very grateful to M. Džamonja, O. Kalenda and W. Marciszewski for inspiring discussions concerning the subject and several helpful comments on the earlier versions of this paper.

2. Corson compact spaces and measures. Let us briefly recall some terminology and notation. For a given cardinal $\kappa$, the Corson space $\Sigma\left(\mathbb{R}^{\kappa}\right)$ is defined as

$$
\Sigma\left(\mathbb{R}^{\kappa}\right)=\left\{x \in \mathbb{R}^{\kappa}:|\{\alpha<\kappa: x(\alpha) \neq 0\}| \leq \omega\right\} .
$$

A topological space $K$ is called a Corson compactum if $K$ is homeomorphic to a compact subset of $\Sigma\left(\mathbb{R}^{\kappa}\right)$ for some $\kappa$.

Given a compact space $K$, we denote by $P(K)$ the set of all probability Radon measures on $K$. As a topological space, $P(K)$ will always be equipped with the weak* topology inherited from $C(K)^{*}$ (and we sometimes write $\mu(g)$ for $\left.\int g d \mu\right)$.

Saying that a given $\mu \in P(K)$ has a separable support, we mean that there is a separable closed $L \subseteq K$ with $\mu(L)=1$. Recall that a measure $\mu$ itself is called separable if $L_{1}(\mu)$ is a separable Banach space. To avoid confusion, we shall say that such a measure $\mu$ is of countable type (cf. [8]).

In general, those two countability conditions for measures are not related. For instance, if $\mu$ is the Lebesgue measure on $[0,1]$, and $\widehat{\mu}$ is the corresponding measure defined on the Stone space of the measure algebra of $\mu$, then $\widehat{\mu}$ is of countable type but has no separable support. On the other hand, the usual product measure on the separable space $\{0,1\}^{\omega_{1}}$ is of type $\omega_{1}$.

Recall that a (finite Borel) measure $\mu$ on a topological space $X$ is said to be strictly positive if $\mu(V)>0$ for every nonempty open set $V \subseteq X$. In the case of a Radon measure $\mu \in P(K)$ this condition simply means that the 
whole space $K$ is "the" support of $\mu$. Chapter 6 of [5] is the basic reference for this subject.

It is well known that every separable Corson compact space is metrizable (see e.g. $[17,6.3(\mathrm{c})]$ ). Hence if a measure $\mu \in P(K)$ on a Corson compactum $K$ has a separable support then $\mu$ is of countable type. Following [1] we say that a Corson compact space $K$ has property (M) if every $\mu \in P(K)$ has a separable support.

Let us recall that it is undecidable in $\mathrm{ZFC}$ if every Corson compact space has property (M). For instance, this is so under Martin's axiom and the negation of $\mathrm{CH}$ (see below). Therefore one cannot expect to have a counterexample to $(*)$ constructed without extra axioms. Several authors presented constructions carried out under $\mathrm{CH}$ (or weaker axioms) of nonseparable Corson compact spaces of weight $\omega_{1}$, and Radon measures defined on them (which can be either of countable or of uncountable type): see Haydon [10], Talagrand [20], Kunen [12], Kunen \& van Mill [13] (cf. [1], [6], [7], $[19])$.

Suppose now that $K$ is a compact convex subset of $\Sigma\left(\mathbb{R}^{\kappa}\right)$. For every $\alpha<\kappa$ we denote by $\pi_{\alpha}$ the usual projection onto the $\alpha$ th coordinate. As noted in [1, p. 215], every $\mu \in P(K)$ has a barycentre point $b_{\mu} \in K$ such that for every $\alpha<\kappa$ we have

$$
\int \pi_{\alpha} d \mu=\pi_{\alpha}\left(b_{\mu}\right)=b_{\mu}(\alpha) .
$$

Indeed, the convexity of $K$ implies the existence of a barycentre of any measure $\mu$ which is a finite combination of Dirac measures, and the compactness implies that every $\mu \in P(K)$ has a barycentre.

The remark above gives short proofs of the following two results; note that the first result is applicable to any compact convex subset of the positive cone $\Sigma\left(\mathbb{R}_{+}^{\kappa}\right)$ of $\Sigma\left(\mathbb{R}^{\kappa}\right)$ (cf. [21, Lemma 9.5]).

THEOREM 2.1. Assume that $K \subseteq \Sigma\left(\mathbb{R}^{\kappa}\right)$ is compact and convex, and has the property that $|x| \in K$ for every $x \in K$. Then every $\mu \in P(K)$ has a separable support.

Proof. Let $a(x)=|x|$; then $a: K \rightarrow K$ is a continuous mapping. Take any $\mu \in P(K)$ and consider the image measure $\nu=a(\mu)$. Then $b_{\nu} \in K$ and the set $I=\left\{\alpha<\kappa: b_{\nu}(\alpha) \neq 0\right\}$ is countable. For $\alpha \notin I$, we have $\pi_{\alpha} \geq 0$ $\nu$-almost everywhere, and $\int \pi_{\alpha} d \nu=b_{\nu}(\alpha)=0$. Hence $\nu\left(\left\{\pi_{\alpha}=0\right\}\right)=1$, and it follows that $\nu$ is concentrated on the metrizable subspace

$$
K_{I}=\{x \in K: x(\alpha)=0 \text { for all } \alpha \in \kappa \backslash I\} .
$$

Consequently, $\mu\left(K_{I}\right)=1$ and the proof is complete.

THEOREM 2.2. Let $K$ be a compact convex subset of $\Sigma\left(\mathbb{R}^{\kappa}\right)$ and let $\mu \in$ $P(K)$. Then $\mu$ is of countable type if and only if it has a separable support. 
Proof. The condition is clearly sufficient since separable Corson compacta are metrizable and every measure on a metrizable compact space is of countable type.

If the measure $\mu$ is of countable type then there is a countable family $\mathcal{C}$ of Borel subsets of $K$ such that for every Borel set $B \subseteq K$ and $\varepsilon>0$ we have $\mu(B \triangle C)<\varepsilon$ for some $C \in \mathcal{C}$. We can assume that $\mu(C)>0$ for every $C \in \mathcal{C}$.

For any $C \in \mathcal{C}$, we denote by $\nu_{C}$ the probability measure defined by the formula $\nu_{C}(\cdot)=\mu(\cdot \cap C) / \mu(C)$. Denote by $b_{C}$ the barycentre of $\nu_{C}$, and let

$$
I=\left\{\alpha<\kappa: b_{C}(\alpha) \neq 0 \text { for some } C \in \mathcal{C}\right\} .
$$

Then $I$ is countable and it suffices to check that $\mu\left(K_{I}\right)=1$ (where $K_{I}$ is defined as in the proof of Theorem 2.1). If $\alpha \in \kappa \backslash I$ then

$$
\frac{1}{\mu(C)} \int_{C} \pi_{\alpha} d \mu=\int \pi_{\alpha} d \nu_{C}=b_{C}(\alpha)=0
$$

for every $C \in C$. It follows that $\int_{B} \pi_{\alpha} d \mu=0$ for every Borel set $B$, since the family $\mathcal{C}$ is $\triangle$-dense. Thus $\pi_{\alpha}=0 \mu$-almost everywhere, and we are done.

Theorem 4.2 presented below shows that there are no obvious generalizations of Theorem 2.1 which would be true in ZFC. It also implies that the statement

(**) $K$ has property $(\mathrm{M})$ if and only if $\operatorname{cl}_{\mathbb{R}^{\kappa}}(\operatorname{conv} K) \subseteq \Sigma\left(\mathbb{R}^{\kappa}\right)$,

where $K \subseteq \Sigma\left(\mathbb{R}^{\kappa}\right)$ is compact, is not provable in ZFC (cf. [1, Corollary 3.6]). (Here and below we write conv $K$ for the convex hull of $K$ and $\operatorname{cl}_{\mathbb{R}^{\kappa}}(A)$ for the closure of $A \subseteq \Sigma\left(\mathbb{R}^{\kappa}\right)$ in $\mathbb{R}^{\kappa}$.) It is interesting to compare (**) with Corollary 2.4 below, which can be derived from Kunen \& van Mill [13] and the following result due to Marciszewski [15].

Theorem 2.3 (Marciszewski). The following are equivalent.

(a) There is a compact $K \subseteq \Sigma\left(\mathbb{R}^{\omega_{1}}\right)$ such that $\operatorname{cl}_{\mathbb{R}^{\kappa}}(\operatorname{conv} K) \supseteq[0,1]^{\omega_{1}}$.

(b) There is a compact $K \subseteq \Sigma\left(\mathbb{R}^{\omega_{1}}\right)$ such that $\operatorname{cl}_{\mathbb{R}^{\kappa}}(\operatorname{conv} K) \nsubseteq \Sigma\left(\mathbb{R}^{\omega_{1}}\right)$.

(c) $\omega_{1}$ is not a precaliber of measure algebras.

Recall that a cardinal number $\kappa$ is a precaliber of a Boolean algebra $\mathfrak{A}$ if for every $\left(a_{\xi}\right)_{\xi<\kappa} \subseteq \mathfrak{A}^{+}$there is $X \subseteq \kappa$ with $|X|=\kappa$ such that $\left(a_{\xi}\right)_{\xi \in X}$ is centred, i.e. $\prod_{\xi \in J} a_{\xi} \neq 0$ for every finite $J \subseteq X$.

Some facts concerning precalibers of measure algebras can be found in Cichoń et al. [4], Cichoń [3] (see also [8], [9], [5]). We need to know that $\omega_{1}$ is a precaliber of all measure algebras if and only if it is a precaliber of the measure algebra of the product measure on $\{0,1\}^{\omega_{1}}$, and the latter is equivalent to saying that $\{0,1\}^{\omega_{1}}$ cannot be covered by $\omega_{1}$ null sets (see $[9$, A2U], cf. [19]). 
Combining the result from Kunen \& van Mill [13] (stating that (b) and (c) below are equivalent) with Theorems 2.2 and 2.3 and the above remark on precalibers we conclude the following.

COROLlary 2.4. The following are equivalent.

(a) Every Corson compact space has property (M).

(b) Every Radon measure on a Corson compactum is of countable type.

(c) $\{0,1\}^{\omega_{1}}$ cannot be covered by $\omega_{1}$ null sets.

(d) $\omega_{1}$ is a precaliber of measure algebras.

(e) $\operatorname{cl}_{\mathbb{R}^{\kappa}}(K) \subseteq \Sigma\left(\mathbb{R}^{\kappa}\right)$ for every $\kappa$ and every compact $K \subseteq \Sigma\left(\mathbb{R}^{\kappa}\right)$.

3. Two 0-dimensional Corson compacta. The spaces constructed below are defined as the Stone spaces of some algebras of sets. If $\mathcal{A}$ is an algebra of subsets of some fixed space then $\operatorname{Ult}(\mathcal{A})$ denotes the Stone space of all ultrafilters on $\mathcal{A}$. For a set $A \in \mathcal{A}$, we denote by $\widehat{A} \subseteq \operatorname{Ult}(\mathcal{A})$ the clopen set associated to $A$ via the usual isomorphism.

We write $\mathcal{A}=\operatorname{alg}(\mathcal{G})$ if $\mathcal{A}$ is the algebra of sets generated by a family $\mathcal{G}$. Observe that if $\mathcal{G}$ is a family with the property that every centred subfamily $\mathcal{G}^{\prime}$ of $\mathcal{G}$ is countable, then $K=\operatorname{Ult}(\mathcal{A})$ is Corson compact. Indeed, the family $\{\widehat{G}: G \in \mathcal{G}\}$ is then point-countable and separating, and the mapping $g(s)=\left(\chi_{\widehat{G}}(s)\right)_{G \in \mathcal{G}}$ is a continuous embedding of $K$ into $\Sigma\left(\mathbb{R}^{\mathcal{G}}\right)$.

By a quasi-measure we mean a nonnegative finitely additive set function defined on an algebra of sets. If $K=\operatorname{Ult}(\mathcal{A})$ then every quasi-measure $\nu$ on $\mathcal{A}$ uniquely defines a Radon measure $\widehat{\nu}$ on $K$, where $\widehat{\nu}(\widehat{A})=\nu(A)$ for every $A \in \mathcal{A}$. In what follows, we denote $\widehat{\nu}$ simply by $\nu$, and it should be clear from the context whether we treat $\nu$ as a quasi-measure on $\mathcal{A}$ or as a Radon measure on $K$.

The construction used in the proof of the theorem given below is a modification of the reasoning from [19, Theorem 5.2(a)], which in turn is an alternative approach to Kunen \& van Mill's result from [13]. Note that we might relax $\mathrm{CH}$ to a weaker axiom (see [19] for details).

TheOREm 3.1. (CH) There are a nonseparable Corson compact space $K$, a strictly positive measure $\mu \in P(K)$ of uncountable type, and a family $\left(g_{\alpha}\right)_{\alpha<\omega_{1}} \subseteq C(K)$ of norm one functions such that for every $\nu \in P(K)$, the set $\left\{\xi<\omega_{1}: \int g_{\xi} d \nu \neq 0\right\}$ is countable.

Proof. We shall work with the usual product measure $\lambda$ defined on the Baire $\sigma$-algebra $\operatorname{Ba}\{0,1\}^{\omega_{1}}$ (which is generated by all clopen subsets of $\left.\{0,1\}^{\omega_{1}}\right)$. Recall that every set $B \in \mathrm{Ba}\{0,1\}^{\omega_{1}}$ is determined by coordinates in some countable set $I \subseteq \omega_{1}$, that is, $B=\pi_{I}^{-1} \pi_{I}(B)$, where $\pi_{I}$ denotes the projection from $\{0,1\}^{\omega_{1}}$ onto $\{0,1\}^{I}$. 
(1) We shall construct a suitable algebra $\mathcal{A}$ contained in $\operatorname{Ba}\{0,1\}^{\omega_{1}}$. This $\mathcal{A}$ will be defined as $\mathcal{A}=\bigcup_{\alpha<\omega_{1}} \mathcal{A}_{\alpha}$, where $\left(\mathcal{A}_{\alpha}\right)_{\alpha<\omega_{1}}$ is an increasing sequence of countable algebras. We let $\mathcal{A}_{0}$ be some countable nonatomic subalgebra of $\mathrm{Ba}\{0,1\}^{\omega_{1}}$, and impose the following requirements:

(i) $\lambda(A)>0$ for every nonempty $A \in \mathcal{A}$;

(ii) $\mathcal{A}_{\alpha}=\operatorname{alg}\left(\bigcup_{\beta<\alpha} \mathcal{A}_{\beta} \cup\left\{H_{\alpha}^{0}, H_{\alpha}^{1}\right\}\right)$, where $H_{\alpha}^{0} \cap H_{\alpha}^{1}=\emptyset, F_{\alpha}:=H_{\alpha}^{0} \cup$ $H_{\alpha}^{1} \neq \emptyset$, and $\lambda\left(C \cap H_{\alpha}^{0}\right)=\lambda\left(C \cap H_{\alpha}^{1}\right)$ whenever $\beta<\alpha$ and $C \in \mathcal{A}_{\beta}$;

(iii) if $\left(A_{n}\right)_{n} \subseteq \mathcal{A}$ is a decreasing sequence with $\lim \lambda\left(A_{n}\right)=0$, then there is $\alpha<\omega_{1}$ such that for all $\eta>\alpha$ there is $n$ such that $A_{n} \cap F_{\eta}=\emptyset$.

(2) We now show how to define the algebras $\mathcal{A}_{\alpha}$ by induction on $\alpha<\omega_{1}$. For every constructed $\mathcal{A}_{\alpha}$ we fix, using $\mathrm{CH}$, an enumeration $\left(s_{\beta}^{\alpha}\right)_{\beta<\omega_{1}}$ of all decreasing sequences $\left(A_{n}\right)_{n} \subseteq \mathcal{A}_{\alpha}$ satisfying $\lim _{n \rightarrow \infty} \lambda\left(A_{n}\right)=0$.

Having $\mathcal{A}_{\eta}$ defined for $\eta<\xi$, write $\mathcal{C}=\bigcup_{\eta<\xi} \mathcal{A}_{\eta}$. First find a set $B \in$ $\operatorname{Ba}\{0,1\}^{\omega_{1}}$ of positive measure such that if $\alpha, \beta<\xi$ then there is $n$ such that $s_{\beta}^{\alpha}(n) \cap B=\emptyset$. The existence of such a set follows from the fact that

$$
\lambda\left(\bigcup_{\alpha, \beta<\xi} s_{\beta}^{\alpha}\left(n_{\alpha, \beta}\right)\right)<\frac{1}{2}
$$

for some choice of natural numbers $n_{\alpha, \beta}$.

Now we define $F_{\xi} \in \mathrm{Ba}\{0,1\}^{\omega_{1}}$ by

$$
F_{\xi}=B \backslash \bigcup\{C \in \mathcal{C}: \lambda(C \cap B)=0\} .
$$

Notice that there is a countable set $I \subseteq \omega_{1}$ such that $F_{\xi}$, as well as every $C \in \mathcal{C}$, is determined by coordinates in $I$. Take any $\gamma \in \omega_{1} \backslash I$; set $D_{\gamma}=$ $\left\{x \in\{0,1\}^{\omega_{1}}: x(\gamma)=0\right\}, H_{\xi}^{0}=F_{\xi} \cap D_{\gamma}, H_{\xi}^{1}=F_{\xi} \backslash D_{\gamma}$. Now if $C \in \mathcal{C}$ then

$$
\begin{aligned}
\lambda\left(C \cap H_{\xi}^{0}\right) & =\lambda\left(C \cap F_{\xi} \cap D_{\gamma}\right)=\lambda\left(C \cap F_{\xi}\right) \lambda\left(D_{\gamma}\right) \\
& =\lambda\left(C \cap F_{\xi}\right) \lambda\left(D_{\gamma}^{\mathrm{c}}\right)=\lambda\left(C \cap F_{\xi} \cap D_{\gamma}^{\mathrm{c}}\right)=\lambda\left(C \cap H_{\xi}^{1}\right),
\end{aligned}
$$

and (ii) follows.

It is clear that if the set $C \cap F_{\xi}$ is nonempty then it has positive measure, and from the above formulas it follows that the sets $H_{\xi}^{i}$ have the same property, so (i) is satisfied.

Finally, the algebra $\mathcal{A}$ satisfies (iii), since for every sequence $\left(A_{n}\right)_{n} \subseteq \mathcal{A}$ as in (iii), there are $\alpha, \beta<\omega_{1}$ such that $\left(A_{n}\right)_{n}=s_{\beta}^{\alpha}$.

(3) Let $K=\operatorname{Ult}(\mathcal{A})$. It follows from (iii) that $K$ is Corson compact (see the remarks at the beginning of this section). The measure $\lambda$ restricted to $\mathcal{A}$ uniquely defines a Radon measure $\mu$ on $K$. Condition (ii) implies that the measure $\mu$ is of uncountable type. Indeed,

$$
\mu\left(C \triangle H_{\xi}^{0}\right)=\mu\left(C \backslash H_{\xi}^{0}\right)+\mu\left(H_{\xi}^{0} \backslash C\right) \geq \mu\left(C \cap H_{\xi}^{1}\right)+\mu\left(H_{\xi}^{1} \backslash C\right)=\mu\left(H_{\xi}^{1}\right)
$$


whenever $C \in \mathcal{A}_{\alpha}$ and $\alpha<\xi$. As $\mu\left(H_{\xi}^{1}\right)>0$, no countable part of $\mathcal{A}$ is $\triangle$-dense in $\mathcal{A}$.

For every $\xi<\omega_{1}$ we define a function $g_{\xi}: K \rightarrow \mathbb{R}$ by the formula

$$
g_{\xi}=\chi_{\widehat{H_{\xi}^{0}}}-\chi_{\widehat{H_{\xi}^{1}}} \text {. }
$$

Clearly $g_{\xi} \in C(K)$, and $\left\|g_{\xi}\right\|=1$.

(4) We claim that for a given compact $G \subseteq K$, if $\mu(G)=0$ then $\widehat{F}_{\xi} \cap G$ $\neq \emptyset$ only for countably many $\xi$.

Indeed, since $G$ is a compact set of measure zero, there is a decreasing sequence $\left(\widehat{A}_{n}\right)_{n}$ of clopen sets such that $G \subseteq \bigcap_{n \in \omega} \widehat{A}_{n}$ and $\lambda\left(A_{n}\right) \rightarrow 0$. Hence the claim follows from (iii).

(5) Now we check that the functions defined in (3) have the required property. This is a consequence of the following two observations.

(i) If a measure $\nu \in P(K)$ is singular with respect to $\mu$, then $\nu\left(\bigcup_{n} G_{n}\right)$ $=1$ for some closed sets $G_{n}$ with $\mu\left(G_{n}\right)=0$. Hence the set $\left\{\xi<\omega_{1}: \int g_{\xi} d \nu\right.$ $\neq 0\}$ is countable by $(4)$.

(ii) Consider a measure $\nu \in P(K)$ which is absolutely continuous with respect to $\mu$, and let $\nu(\cdot)=\int . h d \mu$ for a Borel function $h$ defined on $K$. As $C(K)$ is dense in $L_{1}(\mu)$, there is $\xi<\omega_{1}$ such that for every $\varepsilon>0$ there is a function $h^{\prime}$ of the form

$$
h^{\prime}=\sum_{i \leq k} c_{i} \chi_{\widehat{A}_{i}}, \quad \text { where } A_{i} \in \mathcal{A}_{\xi}, c_{i} \in \mathbb{R},
$$

such that $\int\left|h-h^{\prime}\right| d \mu<\varepsilon$.

We claim that $\int g_{\eta} d \nu=0$ for $\eta>\xi$. Indeed, take any $\eta>\xi$ and consider a function $h^{\prime}$ as above. By (1)(ii) we have $\mu\left(H_{\eta}^{0} \cap A_{i}\right)=\mu\left(H_{\eta}^{1} \cap A_{i}\right)$ for every $i \leq k$, and hence

$$
\int_{\widehat{H_{\eta}^{0}}} h^{\prime} d \mu=\int_{\widehat{H_{\eta}^{1}}} h^{\prime} d \mu, \quad \text { so } \quad \int g_{\eta} h^{\prime} d \mu=0 .
$$

Consequently, $\int g_{\eta} h d \mu=0$ by approximation. But $h$ is the Radon-Nikodym derivative of $\nu$ with respect to $\mu$ so we get $\int g_{\eta} d \nu=\int g_{\eta} h d \mu=0$. This completes the proof of the theorem.

Before proving the next theorem we recall some basic facts concerning extensions of quasi-measures. Let $\nu$ be a quasi-measure on the algebra $\mathcal{A}$ of subsets of $X$. For every $Z \subseteq X$ we write

$$
\nu^{*}(Z)=\inf \{\nu(A): Z \subseteq A \in \mathcal{A}\} \quad \nu_{*}(Z)=\sup \{\nu(A): Z \supseteq A \in \mathcal{A}\} .
$$

Recall that $\nu$ can be extended to a quasi-measure on any algebra $\mathcal{B} \supseteq \mathcal{A}$. In particular, if $\mathcal{B}$ is generated by $\mathcal{A}$ and a set $Z \subseteq X$, then for every 
$c \in\left[\nu_{*}(Z), \nu^{*}(Z)\right]$ there is an extension of $\nu$ to a quasi-measure $\widetilde{\nu}$ on $\mathcal{B}$ with the property that $\widetilde{\nu}(Z)=c$ (see e.g. [14] for further references).

Lemma 3.2. Let $K=\operatorname{Ult}(\mathcal{A})$, and let $M \subseteq P(K)$ be any set. If $\nu \in M$ is a $G_{\delta}$-point in $M$ then there is a countable subalgebra $\mathcal{C} \subseteq \mathcal{A}$ such that if $\nu^{\prime} \in M$ and $\nu^{\prime}(C)=\nu(C)$ for all $C \in \mathcal{C}$ then $\nu^{\prime}=\nu$.

Proof. If $\nu \in M$ is $G_{\delta}$ then there is a countable family $\left(g_{n}\right) \subseteq C(K)$ such that whenever $\nu^{\prime} \in M$ and $\nu^{\prime}\left(g_{n}\right)=\nu\left(g_{n}\right)$ for every $n$ then $\nu^{\prime}=\nu$. Take $\mathcal{C}$ to be a countable algebra in $\mathcal{A}$ with the property that every $g_{n}$ is a uniform limit of a sequence of functions of the form $\sum_{i \leq k} \chi_{\widehat{C}_{i}}$. Then the algebra $\mathcal{C}$ has the required property.

Theorem 3.3. (CH) There is a Corson compact space $K$ and a nonempty weak*-compact convex subset of $P(K)$ such that $M$ has no $G_{\delta}$-points.

Proof. Let $I$ be a countable infinite set such that $I \cap \omega_{1}=\emptyset$, and let $X=I \cup \omega_{1}$. This time we shall work with the usual product measure $\lambda$ on $\{0,1\}^{X}$, defined on the Baire $\sigma$-algebra $\operatorname{Ba}\{0,1\}^{X}$. Given $\xi<\omega_{1}$, we put

$$
D_{\xi}=\left\{x \in\{0,1\}^{X}: x(\xi)=0\right\} .
$$

(1) Denote by $\lambda_{I}$ the usual measure on (the Cantor set) $\{0,1\}^{I}$. Using $\mathrm{CH}$, we can write $\{0,1\}^{I}=\bigcup_{\xi<\omega_{1}} N_{\xi}$, where $\lambda_{I}\left(N_{\xi}\right)=0$, and $N_{\eta} \subseteq N_{\xi}$ for $\eta<\xi<\omega_{1}$. For every $\xi<\omega_{1}$ we find a closed subset $Z_{\xi}$ of $\{0,1\}^{I}$ such that $\lambda_{I}\left(Z_{\xi}\right)>0$ and $Z_{\xi} \cap N_{\xi}=\emptyset$. Note that if $\mathcal{Z}$ is a centred subfamily of $\left\{Z_{\xi}: \xi<\omega_{1}\right\}$ then $Z=\bigcap \mathcal{Z} \neq \emptyset$. Taking any $x \in Z$ we have $x \in N_{\xi}$ for some $\xi<\omega_{1}$, so $\mathcal{Z}$ is necessarily countable.

We put $F_{\xi}=\pi_{I}^{-1}\left(Z_{\xi}\right)$. Then every $F_{\xi}$ is a closed subset of $\{0,1\}^{X}$ with $\lambda\left(F_{\xi}\right)>0$. It is clear that every centred subfamily of $\left\{F_{\xi}: \xi<\omega_{1}\right\}$ must be countable.

(2) For every $\xi<\omega_{1}$, we define a set $H_{\xi} \in \operatorname{Ba}\{0,1\}^{X}$ by $H_{\xi}=F_{\xi} \cap D_{\xi}$. Now we put

$$
\mathcal{H}=\left\{H_{\xi}: \xi<\omega_{1}\right\}, \quad \mathcal{F}=\left\{F_{\xi}: \xi<\omega_{1}\right\}, \quad \mathcal{A}=\operatorname{alg}(\mathcal{F} \cup \mathcal{H}),
$$

and denote by $K$ the Stone space of $\mathcal{A}$. It follows from (1) that $\{\widehat{G}: G \in$ $\mathcal{F} \cup \mathcal{H}\}$ is a point-countable separating family of clopen subsets of $K$, so $K$ is Corson compact.

(3) Denote by $\mu$ the measure $\lambda$ restricted to $\mathcal{A}$ (as well as the corresponding measure from $P(K)$ ). We define $M \subseteq P(K)$ to be the set of those $\nu$ that agree with $\mu$ on $\mathcal{F}$, formally

$$
M=\{\nu \in P(K): \nu(\widehat{F})=\mu(\widehat{F}) \text { for every } F \in \mathcal{F}\} .
$$

Then $M$ is a nonempty compact convex subset of $P(K)$. The fact that $M$ has no $G_{\delta}$-points is a consequence of Lemma 3.2 and the following observation. 
(4) Claim. For every $\nu \in M$, and every countable algebra $\mathcal{C} \subseteq \mathcal{A}$, there is $\nu^{\prime} \in M$ such that $\nu^{\prime} \neq \nu$, while $\nu^{\prime}(C)=\nu(C)$ for $C \in \mathcal{C}$.

Indeed, since $\mathcal{C}$ is countable, there is $\eta<\omega_{1}$ such that $H_{\xi} \notin \mathcal{C}$ for every $\xi \geq \eta$. Fix any $\xi>\eta$, and consider the set $H:=H_{\xi}$. Further let $\sigma$ denote $\nu$ restricted to the algebra $\mathcal{B}=\operatorname{alg}\left(\mathcal{F} \cup\left\{H_{\alpha}: \alpha<\eta\right\}\right)$. Note that $\sigma^{*}\left(D_{\xi}\right)=1$ while $\sigma_{*}\left(D_{\xi}\right)=0$ (since $D_{\xi}$ is "independent" of the domain of $\sigma$ ). Hence

$$
\sigma^{*}(H)=\sigma^{*}\left(F_{\xi} \cap D_{\xi}\right)=\sigma\left(F_{\xi}\right)=\mu\left(F_{\xi}\right)>\sigma_{*}(H)=0 .
$$

By the remarks preceding this theorem we may extend $\sigma$ to a quasi-measure $\nu^{\prime}$ on $\mathcal{A}$ in such a way that $\nu^{\prime}(H) \neq \nu(H)$. Then $\nu^{\prime} \in M$ since $\mathcal{B} \supseteq \mathcal{F}$, and this proves the claim.

Note that in the proof of Theorem 3.3 we used $\mathrm{CH}$ only in (1) to write the Cantor set as a union of $\omega_{1}$ null sets, and hence the theorem holds under this set-theoretic assumption.

4. A counterexample to $(*)$. The proof of the main result of this section is based on Theorem 3.1 and another result on the existence of strictly positive measures. The theorem given below is due to Argyros \& Negrepontis [2]. We enclose here a much shorter proof taken from the unpublished thesis [18] (actually, in what follows we use only (i) $\Rightarrow($ ii)).

THEOREM 4.1. The following are equivalent for a compact space $K$ :

(i) $K$ admits a strictly positive Radon measure;

(ii) the space $\left(P(K)\right.$, weak$\left.^{*}\right)$ admits a strictly positive Radon measure.

Proof. (i) $\Rightarrow$ (ii). Let $\mu \in P(K)$ be strictly positive. For every $n$ put $D_{n}=$ $\left\{r \in[0,1]^{n}: \sum_{i \leq n} r(i)=1\right\}$, and let the mapping $\varphi_{n}: D_{n} \times K^{n} \rightarrow P(K)$ be defined by $\varphi_{n}\left(r, t_{1}, \ldots, t_{n}\right)=\sum_{i \leq n} r(i) \delta_{t_{i}}$.

For every $n$ the space $D_{n} \times K^{n}$ has a strictly positive probability Radon measure $\mu_{n}$ (see $[5$, Chapter 6$]$ ). Since every $\varphi_{n}$ is continuous, and the set $\bigcup_{n} \varphi_{n}\left(D_{n} \times K^{n}\right)$ is weak ${ }^{*}$-dense in $P(K)$, it follows that the Radon measure $\Upsilon=\sum_{n=1}^{\infty} 2^{-n} \varphi_{n}\left(\mu_{n}\right)$ is strictly positive on $P(K)$.

(ii) $\Rightarrow($ i). Suppose that $\Upsilon$ is a strictly positive probability Radon measure on $P(K)$. Then the formula

$$
\theta(g)=\int_{P(K)} g(\nu) d \Upsilon(\nu)
$$

where $\nu(g)=\int g d \nu$, defines a positive functional on $C(K)$ with $\|\theta\|=1$. By the Riesz theorem $\theta$ is represented by some $\mu \in P(K)$, which is easily seen to be strictly positive.

Now we are ready to prove the result announced in the introduction. 
THEOREM 4.2. (CH) There exist a compact convex subspace L of $\Sigma\left(\mathbb{R}^{\omega_{1}}\right)$, and a measure $\lambda \in P(L)$ which has no separable support (and, moreover, is of uncountable type).

Proof. Let $K$ and $\left(g_{\xi}\right)_{\xi<\omega_{1}}$ be as in Theorem 3.1. Then

$$
\Phi: P(K) \rightarrow \Sigma\left(\mathbb{R}^{\omega_{1}}\right), \quad \Phi(\nu)=\left(\int g_{\xi} d \nu\right)_{\xi<\omega_{1}},
$$

is an affine continuous mapping, and hence $L=\Phi(P(K))$ is a compact convex subset of $\Sigma\left(\mathbb{R}^{\omega_{1}}\right)$. It is clear that $L$ is nonseparable. Indeed, for every $\xi<\omega_{1}$, taking any $t_{\xi} \in K$ with $g_{\xi}\left(t_{\xi}\right)=1$, we have $\Phi\left(\delta_{t_{\xi}}\right)(\xi)=1$.

Now $P(K)$ has a strictly positive Radon measure by Theorems 4.1 and 3.1 ; suppose $\Upsilon$ is such a measure. Then the image measure $\lambda=\Phi(\Upsilon)$ is strictly positive and Radon on $L$, and thus $\lambda$ has no separable support (and $\lambda$ is of uncountable type by Theorem 2.2).

We remark that in Theorem 4.1 we may replace $P(K)$ by the unit ball of $C(K)^{*}$. Consequently, the space $L$ from Theorem 4.2 can be taken to be absolutely convex.

As mentioned above, the measure $\lambda$ in Theorem 4.2 is of uncountable type. Note that writing $L_{\xi}=\{x \in L: x(\xi) \geq 1 / 2\}$ we have an uncountable family $\left(L_{\xi}\right)_{\xi<\omega_{1}}$ of compact convex sets of positive measure with the property that none of its uncountable subfamilies has a nonempty intersection. It is plausible that Theorem 2.2 can be generalized to saying that such a family can exist for no Radon measure of countable type which is defined on a locally convex vector space.

It should be stressed that the space $P(K)$ itself, where $K$ is the space from Theorem 3.1, is not Corson compact (since $P(K)$ is not FréchetUrysohn; cf. [11] for other topological properties of such spaces). This implies that the mapping $\Phi$ considered above cannot be injective. O. Kalenda observed that, based on Theorem 3.5 from [1] and Theorem 2.1 above, we can conclude the following.

THEOREM 4.3. For a compact $K$ the following are equivalent:

(a) $K$ is Corson compact and has property (M);

(b) $P(K)$ is Corson compact;

(c) $P(K)$ is Corson compact and has property (M);

(d) $B_{C(K) *}$ is Corson compact;

(e) $B_{C(K)^{*}}$ is Corson compact and has property (M).

So for a Banach space of the form $C(K)$, the dual unit ball $B_{C(K)^{*}}$ has property $(\mathrm{M})$ provided it is Corson compact at all. On the other hand, we have the following. 
Corollary 4.4 (Kalenda). Under $C H$, there is a Banach space E such that the dual unit ball $B_{E^{*}}$ is a Corson compact space without property $(\mathrm{M})$.

Proof. In the notation of Theorem 3.1, let $E$ be the closed subspace of $C(K)$ spanned by the family $\left(g_{\alpha}\right)_{\alpha<\omega_{1}}$. Write $B=B_{E^{*}}$ for the dual unit ball; it is the image of $B_{C(K)^{*}}$ under the natural restriction map $r$ : $B_{C(K)^{*}} \rightarrow B$. We can use again the mapping $\Phi: B_{C(K)^{*}} \rightarrow \Sigma\left(\mathbb{R}^{\omega_{1}}\right)$, where $\Phi(\sigma)=\left(\sigma\left(g_{\xi}\right)\right)_{\xi<\omega_{1}}$, and $\sigma\left(g_{\xi}\right)$ is the integral with respect to a signed Radon measure $\sigma$. Define $f: B \rightarrow \Sigma\left(\mathbb{R}^{\omega_{1}}\right)$ by $f(r(\sigma))=\Phi(\sigma)$. Then $f$ is an affine embedding of $B$ into $\Sigma\left(\mathbb{R}^{\omega_{1}}\right)$, so $B$ is Corson compact. Note that $f(B)$ contains the space $L$ from Theorem 4.2 so neither $f(B)$ nor $B$ has property $(\mathrm{M})$.

\section{References}

[1] S. Argyros, S. Mercourakis and S. Negrepontis, Functional analytic properties of Corson-compact spaces, Studia Math. 89 (1988), 197-229.

[2] S. Argyros and S. Negrepontis, Chain conditions for spaces of measures, preprint, 1982.

[3] J. Cichoń, On two cardinal properties of ideals, Trans. Amer. Math. Soc. 314 (1989), 693-708.

[4] J. Cichoń, A. Szymański and B. Węglorz, On intersections of sets of positive Lebesgue measure, Colloq. Math. 52 (1987), 173-177.

[5] W. W. Comfort and S. Negrepontis, Chain Conditions in Topology, Cambridge Univ. Press, 1982.

[6] M. Džamonja and K. Kunen, Measures on compact HS spaces, Fund. Math. 143 (1993), 41-54.

[7] - - - Properties of the class of measure separable compact spaces, ibid. 147 (1995), 261-277.

[8] D. H. Fremlin, Measure algebras, in: Handbook of Boolean Algebras, J. D. Monk (ed.), North-Holland, 1989, Vol. III, Chap. 22.

[9] -, Real-valued measurable cardinals, in: Israel Math. Conf. Proc. 6, Bar-Ilan Univ., 1993, 151-304.

[10] R. Haydon, On dual $L^{1}$-spaces and injective bidual Banach spaces, Israel J. Math. 31 (1978), 142-152.

[11] O. Kalenda, A characterization of Valdivia compact spaces, Collect. Math. 51 (2000), 59-81.

[12] K. Kunen, A compact L-space under CH, Topology Appl. 12 (1981), 283-287.

[13] K. Kunen and J. van Mill, Measures on Corson compact spaces, Fund. Math. 147 (1995), 61-72.

[14] Z. Lipecki, On unique extensions of positive additive set functions, Arch. Math. (Basel) 41 (1983), 71-79.

[15] W. Marciszewski, Convex hulls of Corson compact spaces, preprint, 1984.

[16] S. Mercourakis and S. Negrepontis, Banach spaces and topology, in: Recent Progress in General Topology, M. Hušek and J. van Mill (eds.), North-Holland, 1992, 493-536.

[17] S. Negrepontis, Banach spaces and topology, in: Open Problems in Topology, J. van Mill and G. M. Reed (eds.), Chap. 23. 
[18] G. Plebanek, On strictly positive measures and $\tau$-additive measures, doctoral dissertation, Univ. of Wrocław, 1990.

[19] —, Nonseparable Radon measures and small compact spaces, Fund. Math. 153 (1997), 25-40.

[20] M. Talagrand, Séparabilité vague dans l'espace des mesures sur un compact, Israel J. Math. 37 (1980), 171-180.

[21] S. Todorčević, Chain condition methods in topology, Topology Appl. 101 (2000), $45-82$.

Institute of Mathematics

University of Wrocław

Pl. Grunwaldzki 2/4

50-384 Wrocław, Poland

E-mail: grzes@math.uni.wroc.pl

Received 11 February 2002;

in revised form 20 June 2002 